\title{
Sound user Interface with Touch Panel for Data and Information Expression and its Application to Meteorological Data Representation
}

\author{
Kohei Arai \\ Graduate School of Science and Engineering, Saga University, Saga City, Japan
}

\begin{abstract}
Sound User Interface (SUI) with touch panel for representation of quantitative data and information together with its application to meteorological data representation is proposed. The proposed SUI is not a merely ear-con. Through experiments, it is found that the proposed SUI combined with visual perception makes meteorologist to understand meteorological data intuitively and is much understandable than ever in a comprehensive manner. It is also useful to hear "images" in particular, for blind person.
\end{abstract}

Keywords-Audible; meteorology; remote sensing satellite; musical scale; multi-layered data; SUI

\section{INTRODUCTION}

Recently, new user Interface: UI is invented and developed. Tangible Interface (TI) ${ }^{1}$ is one of those. TI allows users touch data or information. It differs from touch interface. Touch interface allows caution/warning/awareness signs and mouse operations with touching "Touch Screen"2. On the other hand, the current Sound Unser Interface: SUI allows caution/warning/awareness signs and mouse operations no more than that. New SUI proposed here which allows not only caution/warning/awareness signs and mouse operations but also hear/listen data and information is my concern.

In the Apple Watch Series 3 and $4^{3}$ released in September 2017 and quite recently, respectively, the voice command by Siri ${ }^{4}$ has been further strengthened. Sound-based communication may become more important than ever. Recently, we have been surrounded by enormous amount of information which has not existed so far. Originally, humans acquire information by using various information about surroundings using five senses, but most information on current information equipment is displayed by visual media. However, in recent years, users' experiences in applications and Web services attracted attention in the user interface, UI of SUI which makes use of non-verbal sounds in addition to visual UI plus sound, especially voice.

One major problem emerges as the downsizing of equipment such as smart phones and wearable devices is accelerated. It means that there is a limit to the size of the

\footnotetext{
${ }^{1}$ https://en.wikipedia.org/wiki/Tangible_user_interface

${ }^{2}$ https://en.wikipedia.org/wiki/Touchscreen

${ }^{3}$ https://www.apple.com/jp/apple-watch-series4/?afid=p238\%7CLoYxmRFF-dc_mtid_2092567642642\&cid=wwa-jp-kwyhwatch

${ }^{4}$ https://ja.wikipedia.org/wiki/Siri
}

visual display and the purpose of keeping staring at the display gradually diminishes. Due to miniaturization of devices, information display means to replace visual information has come to be desired. Actually, many of us have heard the sound as a UI. For example, on desktops such as Windows and MacOS, if you empty the trashcan, you will hear the "Crash sound". If this sound does not occur, the user does not know whether the trash can really be empty, once you open the trash can. However, as the sound is steadily generated, the user can be confident that the action of emptying the trash box has been completed.

The communication application such as LINE $^{5}$ and Facebook Messenger ${ }^{6}$ also implements a function to notify that a message has been received by sound. This allows you to confirm that the message arrived instantaneously by picking up the sound even if the user is doing another work.

SUI has some advantages over visual UI, so it is important to use it effectively. Here let's examine the features of SUI and the three elements necessary to design SUI. The SUI is to convey some information, and there are messages (information) to be displayed there.

Sonification system for representation of satellite remote sensing data is proposed [1] together with sonification method for representation of multi-dimensional meteorological data derived from Earth observation satellites [2]. Recently, method for audible representation of meteorological data derived from remote sensing satellites is proposed [3]. Next section describes some related research together with the proposed method followed by experiments with atmospheric sounder data derived from remote sensing satellites as an example. Finally, some concluding remarks and discussions are described.

\section{RELATED RESEARCH}

$\mathrm{SUI}^{7}$ is not so popular in the user interface research field. Adequate context for interpreting audibles of data is not so easy [4]. Also, meaning is many audible attempts are coded from scratch [5]. The opto-phone which consists of selenium photo-sensors to detect black print and convert it into an audible output was invented [6]. The first experiment of the transmission of information via auditory display was

\footnotetext{
${ }^{5}$ https://line.me/ja/

${ }^{6} \mathrm{https}: / / \mathrm{www}$. facebook.com/messenger

${ }^{7} \mathrm{http}: / /$ sounduserinterface.org/sui/
} 
published [7]. The "Auditory Data Inspection (ADI)" is proposed and reported in the technical memorandum [8]. The effectiveness of the ADI has not been done yet [9]. Another audible system, so called "Pulse Oximeter" which allows audible oxygen concentration of blood was reported [10]. International Community for Auditory Display (ICAD) ${ }^{8}$ has been established and its conferences have been conducted [11]. There are some interactive audible techniques [12-14]. ModelBased Audible, Parameter Mapping Audible, and StreamBased Audible are identified as difficulties [15].

SUI proposed here is the interface with touch panel for representation of quantitative data and information together with its application to meteorological data representation. The proposed SUI is a brand new method which allows hearing the satellite data as well as meteorological data. Through experiments, it is found that the proposed SUI combined with visual perception makes meteorologist to understand meteorological data intuitively and is much understandable than ever in a comprehensive manner. It is also useful to hear "images" in particular, for blind person.

\section{PROPOSED METHOD}

\section{A. Design Concept of the Proposed SUI}

These sound data are created by using "Sakura" software tool $^{9}$ (Free open source software for sound representation). It provides sound sources with the different music instrument types, musical scale, rhythm, loudness, harmony, etc. with text representations.

\section{B. Detailes of Sakura}

"Sakura" allows easily compose with" katakana" character such as "ドレミファソラシド: Doremifa solasido" representing the scale. In the built-in text editor, it is possible to quickly input symbols such as sharp, flat, octave raising and lowering from the list. Musical Instrument Digital Interface ${ }^{10}$ (MIDI) type of output files can be created together with Windows Media Player ${ }^{11}$ (WMP) type files. After composing, it is possible to play with MIDI, save it as a text file or MIDI file. It is possible to also play songs created with other text editors or songs sent by email from friends. In addition to the katakana scale, it also supports MML $^{12}$ which is widely used in computer music.

Since it has a script function, it can also extend its functions such as delicate musical expression and algorithmic composition. It is possible to also listen to lots of songs made by users on the author WEB page. For example, it is possible to play a tulip with "Dreme Remy Mile Dre Mile". Sakura is developed as a foundation of Music Macro Language ${ }^{13}$ (MML). MML is a musical notation language devised to describe the Back Ground Music (BGM) of the game by expressing Doremifa solasi as "cdefgab".

\footnotetext{
${ }^{8}$ http://www.icad.org/

${ }^{9} \mathrm{https} / / /$ forest.watch.impress.co.jp/library/software/sakura/

${ }^{10} \mathrm{https} / / /$ ja.wikipedia.org/wiki/MIDI

${ }^{11}$ https://ja.wikipedia.org/wiki/Windows_Media_Player

${ }^{12} \mathrm{https}: / /$ ja.wikipedia.org/wiki/MMI

${ }^{13}$ https://ja.wikipedia.org/wiki/Music_Macro_Language
}

Also, by writing a simple script in the song, there is also the merit that it is possible to expand its function. If you use Sakura, it is possible to make music of any genre from classical to pop, finally to experimental music, with a sense of word processor.

Sakura has a scripting function that allows you to use control structures such as If and While. If you enjoy music all the way to a senior class, try using the script, algorithm composition! Also, it is the real pleasure of Sakura that it is possible to enjoy music of the source like maniacal, such as concept music, modern music, experimental music and random number music.

Fig. 1 shows the initial displayed screen shot. All the available functions are aligned at the top row on the other hand, operation (or functional) menus are aligned at the left column.

Fig. 2 shows the operational display divisions. There are six divisions, text editor, keyboard input, enter staff notes, useful tab (functional menu), message display, and play monitor.

Detailed operations are as follows:

1) Text editor: Write performance information such as "Doremi" in the text editor.

2) Button for stop the play: Press the playback button.

3) Message display: If there is a mistake in the performance information, the message will be displayed in.

4) Play monitor: The performance monitor is displayed and the performance starts.

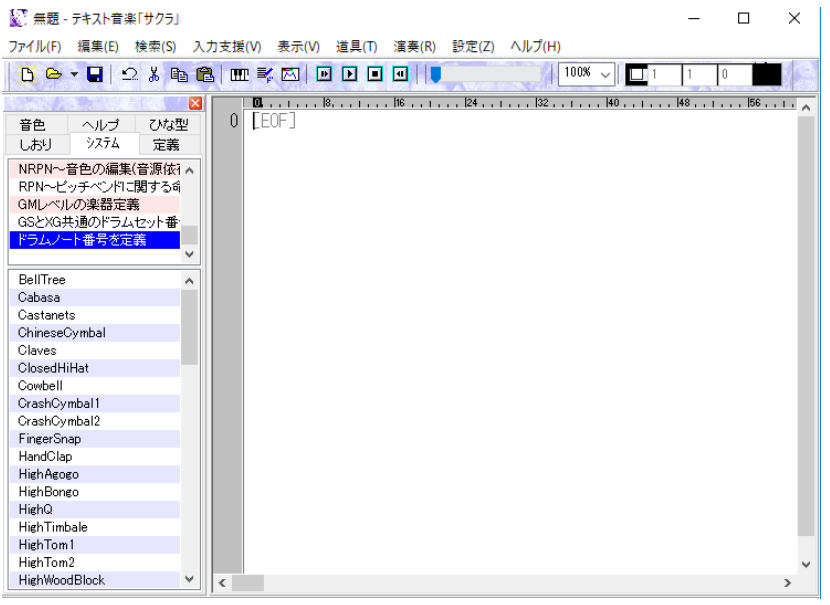

Fig. 1. Initial Display of Screen Shot.

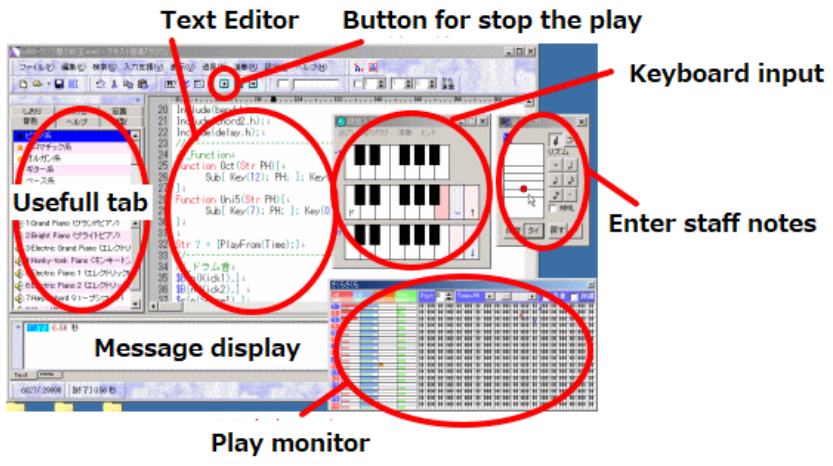

Fig. 2. Display Screen Divisions. 
It is a colorful performance monitor, parameters per channel on the left side. The keyboard on the right side is colored with the key being pressed. It is possible to customize the color. When you click the keyboard or staff score with the mouse, the performance information of Doremi is inserted in the editor. In case of staff input, to enter sharp, press the [SHIFT] key.

1) Message tab: Messages, help, etc. are displayed.

2) Keyboard input: It is possible to insert Doremi text by clicking the keyboard indicated by the picture.

3) Score entry: It is possible to insert Doremi text by clicking on the staff.

4) Convenient tab: It is possible to watch the tone and insert instructions.

5) Bookmark: It is possible to set bookmarks in any portion of the editor.

By using simple bookmarks, it is possible to set bookmarks at arbitrary points (up to 10 places) and instantly return to that place. As for the method of setting simple bookmarks, when you right-click on the editor, the menu "register bookmark" appears, so it is possible to set bookmarks by selecting the registration number. It also supports shortcuts (same as Borland Delphi ${ }^{14}$ ), with Shift + Ctrl + Numbers, it is possible to go back to the bookmark with Bookmark Registration and Ctrl + Numbers.

The bookmark function is located on the command insertion tab, giving a name to arbitrary parts of the editor, so that it is possible to jump to that part immediately afterwards. To create a bookmark with the bookmark function, write a comment such as "// _ name" at the beginning of the line of the editor, in the editor, or right click on the editor, it comes out "bookmark It is possible to also do it using "Register".

The circulation function of song bulletin board; it is possible to quickly download and listen to user-created songs. Text music "Sakura" has been published on the Internet since November 5, 1999. The number of users has also increased. And, above all, many wonderful users are using Sakura to make songs. On February 12, 2002, as a place for exchanging users of Sakura, let's show off songs made with Sakura! For the purpose of being, a song bulletin board 3 was set up (* Older song bulletin board has been abolished since many works with problems in copyright were posted.).

1) From the toolbar above the editor, select the icon shown in Fig. 3.

2) Download the latest list. Since songs are frequently posted on the song bulletin board, select "Download latest list from WEB" from the "Download" menu as shown in Fig. 4.

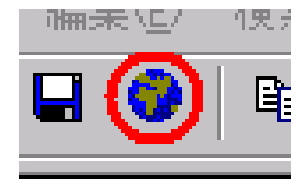

Fig. 3. Selected Icon in the Toolbar.

\footnotetext{
${ }^{14}$ https://ja.wikipedia.org/wiki/Delphi
}

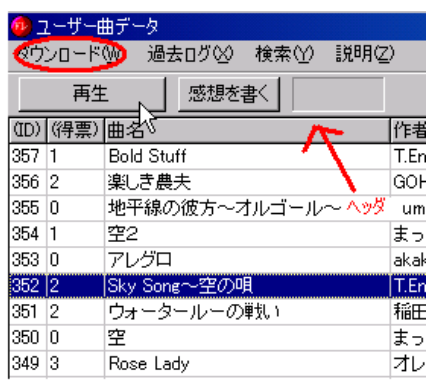

Fig. 4. Download Menu in the Toolbar.

3) To listen to the song, move the cursor to the song title and press the [Play] button. At this time, by clicking on "header" at the top of the list, it is possible to rearrange the data in order of vote, song title, and author. In the song message board, if the number of songs posted exceeds 200 songs, the first 100 songs will move to the "past log". There are many wonderful songs among the songs that have moved to the past log. Therefore, if you select [Obtain past log list from WEB] from the past log menu, a list of past logs is displayed. If you select the past log from among them, it is possible to listen to the past log songs.

This software (Sakura) has a function to check this songboard bulletin board. It is nice feeling that the songs you make are adopted in games, etc. It is natural that people who cannot make music wish to add background music to the game. In this bulletin board, "I cannot make music but I want to add music as a homepage or BGM of the game!", "I use Sakura made songs as BGM, as a game, a website, etc. as BGM A MIDI material bulletin board as a place to interact with each other is desired.

MIDI material bulletin board MIDI data can be easily registered to the MIDI material bulletin board. help.

Quick help Double-click a word on the editor to display

In the editor of Sakura, when you double-click a word (or press the F1 key) with a mouse, the status bar shows how to use the command. For example, if you write Track on the editor, double-click the word with the mouse, or press F1 key, the word part is selected and quick help is displayed in the status bar. When help is displayed, if you want to see more detailed explanation, please click the status bar. Then, detailed help is displayed. Also, when you click on a variable, etc. the number of lines in which the variable is defined is displayed. At this time, clicking the status bar causes the cursor to jump to the variable definition line.

Control with DDE is possible to operate the Sakura editor from plugins and so on.

1) Use $D D E$ in editor: If a language of which it is possible to use Dinamic Data Exchange (DDE) ${ }^{15}$ that Windows uses for communication of applications. It is also possible to use Sakura.exe, a cherry editor, as a DDE reception (server).

\footnotetext{
${ }^{15}$ https://en.wikipedia.org/wiki/Dinamic_data_exchange
} 
2) Benefits: By operating the editor of Sakura from the program, it is possible to easily perform fixed form processing such as inserting and replacing sentences.

3) How to use: Here, we introduce an example of editor operation using my Japanese program language "Sunflower". To send a command to the Sakura Editor on a sunflower, use the instruction "send DDE". For example, to save the program you are currently editing, send "DDE" to "Sakura", "command", "save". I will write it.

(Rewrite the inside of the third "" to the following command.)

new: Initialize the editor

open File name: Opens the file specified in the file name.

save: Save the file currently being edited.

saveas file name: Save the program being edited to the file specified in the file name.

Insert string: Inserts a string at the current cursor position. row Line number: Move the cursor to the line number.

col Column number: Move the cursor to the column number.

copy: Copies the currently selected text to the clipboard.

paste: Paste the contents of the clipboard into the editor.

seltext_save file name: Save the selected text to the specified file.

seltext_open file name: Rewrite the selected text to the contents of the specified file.

play: Play the contents of the editor.

stop: Stops playing.

Sample: "Replace selected text"

The following sample is a program that converts the part selected by the editor to capital letters. Selecting editor Transform text to uppercase:

DDE-transmit "copy" to "command" of "Sakura". '*** 1

Choose "Make text uppercase". If it is not, it will be ended.

Open clipboard. It converts it to uppercase. '*** 2

Save it to the clipboard. DDE sends "paste" to "command" of "himapad". '*** 3

end

*** 1: Instruct the cherry editor to copy the selection area to the clipboard.

*** 2: Convert the contents of the clipboard to uppercase

$* * * 3$ : Instruct the editor of Sakura to paste the contents of the clipboard to the selection area.

\section{EXPERIMENT}

\section{A. Conversion from Meteorological Data to Sound Data}

Atmospheric pressure, wind direction, wind speed at the different altitude can be converted to numerical data as shown in Fig. 5. This is an example of text data of wind speed as function of time and atmospheric pressure. This is same things for the other meteorological data, wind direction, air temperature, relative humidity, etc. Thus, all kinds of meteorological data are represented as numerical text data. Then, these text data are converted to sound data of musical scale (melody), harmony, loudness, rhythm music instrument types as shown in Fig. 6. This is just an example for wind speed at the different altitude, or designated atmospheric pressure.

These are recorded on the track 1-4 depending on the altitude with the different music instrument types. On the other hand, wind direction is represented with musical scale (melody) depending on the eight different wind directions.

Sakura makes the following different types of sound features:

Rhythm, musical instrument type, melody, loudness,

Therefore, atmospheric pressure, air temperature, relative humidity, wind direction can be represented by input the text which is corresponding to the aforementioned meteorological data. Four different types of text input to Sakura are shown in Fig. 7. Thus the proposed system based on Sakura would help meteorologists for comprehensive understanding of meteorological and weather data and information in particular for weather forecasting.

This is just an example. There are so many other data and information which has to be represented by at least five data and information simultaneously.

\begin{tabular}{|c|c|c|c|c|c|c|c|}
\hline \multicolumn{2}{|c|}{$300 \mathrm{hPa}$} & \multicolumn{2}{|c|}{$500 \mathrm{hPa}$} & \multicolumn{2}{|c|}{$700 \mathrm{hPa}$} & \multicolumn{2}{|c|}{$850 \mathrm{hPa}$} \\
\hline Time W & Find & Time & ind & Time $\mathrm{V}$ & Find & Time & Find \\
\hline 6:00 & 1 & 6:00 & 1 & 6:00 & 1 & 6:00 & 1 \\
\hline 0 & & 5 & & 0 & & 8 & \\
\hline 7:00 & 1 & $7: 00$ & 1 & 7:00 & 1 & 7:00 & 1 \\
\hline 2 & & 7 & & 4 & & 6 & \\
\hline 8:00 & 1 & 8:00 & 1 & 8:00 & 1 & 8:00 & 1 \\
\hline 3 & & 8 & & 5 & & 5 & \\
\hline 9:00 & 1 & 9:00 & 2 & 9:00 & 1 & 9:00 & 1 \\
\hline 5 & & 0 & & 8 & & 5 & \\
\hline $10: 00$ & 1 & $10: 00$ & 2 & $10: 00$ & 1 & $10: 00$ & 1 \\
\hline 7 & & 2 & & 9 & & 7 & \\
\hline
\end{tabular}

Fig. 5. Numerical Data of Atmospheric Pressure, Wind Direction, and Wind Speed.

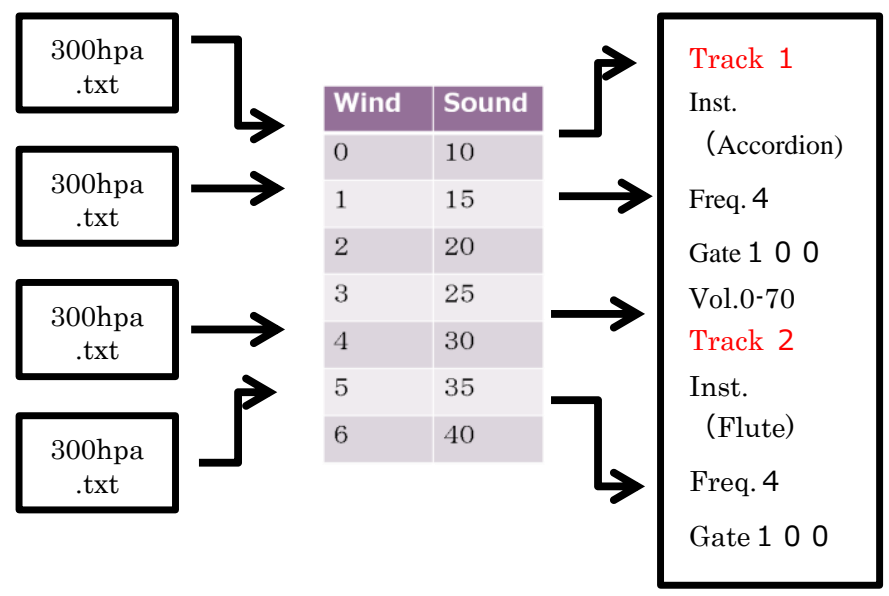

Fig. 6. Conversion from Text File to Sound Data 


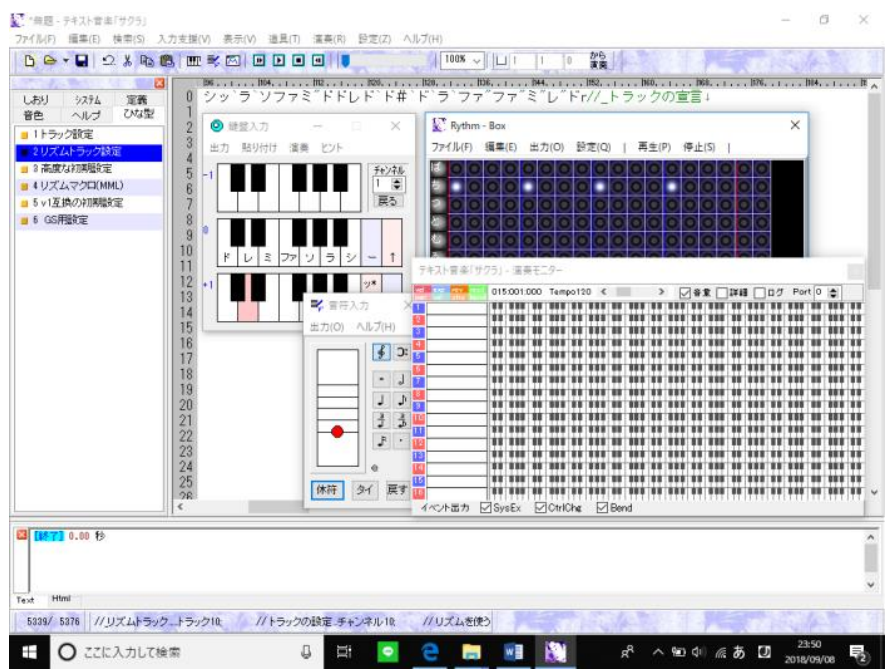

Fig. 7. Four Different Types of Meteorological Data Input to Sakura

\section{B. Examples of Meteorological Satellite Data}

Fig. 8(a) shows the edited sound data referring to text data which are converted from the TOVS/HIRS and MSU: Microwave Sounding Unit $^{16}$ derived meteorological data for September 11 to 22 in 2006 data while Fig. 8(b) shows those for December 1 to 12 in 2011. Although it is difficult to represent the sounds for both examples of meteorological data with this paper, it is confirmed that calm sound for December data while busy sound for September data. Also, it is confirmed the sounds of 3D meteorological data of air temperature, atmospheric pressure, relative humidity, wind direction and wind speed with the different music instrument types.

Values of meteorological data can be represented as volume (loudness) while the altitude can be represented as musical scale. The inverse relation between altitude and air temperature, relative humidity, atmospheric pressure can be identified with the sound.

Thus, user can hear the air temperature profile, relative humidity profile, atmospheric pressure of the pixel location in the MTSAT: Multi-functional Transport Satellite ${ }^{17}$ image, for instance, displayed onto touch screen display by touching the pixel in concern. Most of meteorologist can understand the meteorological situation by location by location, it is hard to see the situation using just visual perception of the vertical profile images displayed onto screen though.

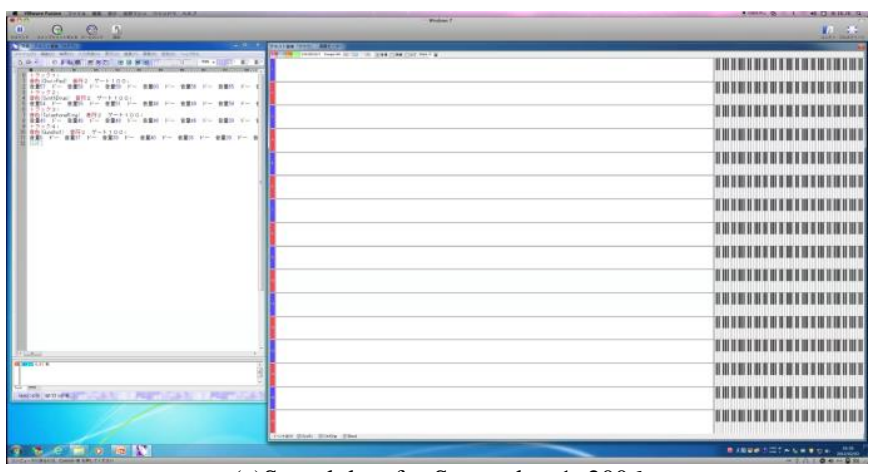

(a)Sound data for September 1, 2006

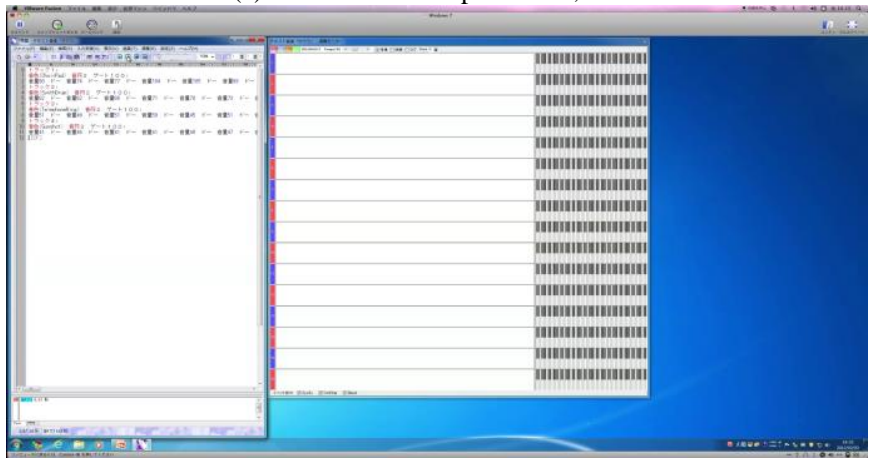

(b)Sound data for December 1, 2011

Fig. 8. Edited Sound Data Referring to Text Data which are Converted from the TOVS ${ }^{18} /$ HIRS $^{19}$ and MSU ${ }^{20}$ Derived Meteorological Data.

\section{CONCLUSION}

Sound User Interface: SUI with touch panel for representation of quantitative data and information together with its application to meteorological data representation is proposed. The proposed SUI is not a merely ear-con. Through experiments, it is found that the proposed SUI combined with visual perception makes meteorologist to understand meteorological data intuitively and is much understandable than ever in a comprehensive manner. It is also useful to hear "images" in particular, for blind person.

It is confirmed that the proposed SUI allows representation of meteorological data, air temperature, atmospheric pressure, relative humidity, wind direction and wind speed.

Further research works are required to expand the application fields of the proposed combined representation of weather data derived from remote sensing satellites. Also, application of the proposed SUI method will be for the blind persons.

\footnotetext{
${ }^{16}$ https://ja.wikipedia.org/wiki/TIROS-N/NOAA

${ }^{17} \mathrm{https} / / /$ ja.wikipedia.org/wiki/MTSAT
}

\footnotetext{
${ }^{18} \mathrm{https} / / /$ www.ozonelayer.noaa.gov/action/tovs.htm

${ }^{19} \mathrm{https} / / /$ ja.wikipedia.org/wiki/TIROS-N/NOAA

${ }^{20} \mathrm{https} / / /$ www.weblio.jp/wkpja/content/TIROS-N/NOAA_TIROSN/NOAA の概要
} 


\section{ACKNOWLEDGMENT}

The author would like to thank Mr. Tsuyoshi Morita of Saga University for his effort to conduct the experiments.

\section{REFERENCES}

[1] Shinichi Sobue, Kohei Arai, Hayato Okumura, Aya Yamamoto, Hiroshi Araki, Tsuneo Matsunaga, Proposed sonification system for representation of satellite remote sensing data, Journal of Space Research Information Analysis Society of Japan, 2011

[2] Kohei Arai, Sonification method for representation of multi-dimensional meteorological data derived from Earth observation satellites, International Journal of Research and Review on Computer Science, 3, 2, 1538-1542, 2012.

[3] Kohei Arai, Method for audible representation of meteorological data derived from remote sensing satellites, Proeedings of the SAI IntelliSys Conefrence 2018, to be published in the Springer Book Series, 2018.

[4] Kramer, Gregory, ed. (1994). Auditory Display: Audible, Audification, and Auditory Interfaces. Santa Fe Institute Studies in the Sciences of Complexity. Proceedings Volume XVIII. Reading, MA: AddisonWesley. ISBN 0201626039.

[5] Flowers, J. H. (2005), Brazil, Eoin, ed., "Thirteen years of reflection on auditory graphing: Promises, pitfalls, and potential new directions", Proceedings of the 11th International Conference on Auditory Display (ICAD2005): 406-409, http://www.icad.org/Proceedings/2005/ Flowers2005.pdf

[6] d'Albe, E. E. Fournier (May 1914), "On a Type-Reading Optophone", Proceedings of the Royal Society of London

[7] Pollack, I. and Ficks, L. (1954), "Information of elementary multidimensional auditory displays", Journal of the Acoustical Society of America

[8] Chambers, J. M. and Mathews, M. V. and Moore, F. R. (1974), "Auditory Data Inspection", Technical Memorandum 74-1214-20

[9] Frysinger, S. P. (2005), Brazil, Eoin, ed., "A brief history of auditory data representation to the 1980s", Proceedings of the 11th International Conference on Auditory Display (ICAD2005) (Department of Computer Science and Information Systems, University of Limerick): 410-413, http://www.icad.org/Proceedings/2005/Frysinger2005.pdf

[10] "Continuous auditory monitoring--how much information do we register?", British Journal of Anaesthesia 83 (5): 747-749, 1999, doi:10.1093/bja/83.5.747, http://bja.oxfordjournals.org/content/83/5/74 7.full.pdf
[11] Kramer, G. and Walker, B.N. (2005), "Sound science: Marking ten international conferences on auditory display", ACM Transactions on Applied Perception (TAP) 2 (4): 383-388, http://citeseerx.ist.psu.edu/viewdoc/download?doi=10.1.1.88.7945\&rep $=$ rep $1 \&$ type $=$ pdf

[12] Thomas Hermann, Andy Hunt, and Sandra Pauletto. Interacting with Audible Systems: Closing the Loop. Eighth International Conference on Information Visualisation (IV'04) : 879-884. Available: online. DOI= http://doi.ieeecomputersociety.org/10.1109/IV.2004.1320244.

[13] Thomas Hermann, and Andy Hunt. The Importance of Interaction in Audible. Proceedings of ICAD Tenth Meeting of the International Conference on Auditory Display, Sydney, Australia, July 6-9, 2004. Available: online

[14] Sandra Pauletto and Andy Hunt. A Toolkit for Interactive Audible. Proceedings of ICAD Tenth Meeting of the International Conference on Auditory Display, Sydney, Australia, July 6-9, 2004. Available: online.

[15] Stephen Barrass. Developing the Practice and Theory of Stream-based Audible. Journal of Media Arts Culture, scan, Available: online

\section{AUTHOR'S PROFILE}

Kohei Arai: He received BS, MS and PhD degrees in 1972, 1974 and 1982, respectively. He was with The Institute for Industrial Science and Technology of the University of Tokyo from April 1974 to December 1978 also was with National Space Development Agency of Japan from January, 1979 to March, 1990. During from 1985 to 1987, he was with Canada Centre for Remote Sensing as a Post Doctoral Fellow of National Science and Engineering Research Council of Canada. He moved to Saga University as a Professor in Department of Information Science on April 1990. He was a councilor for the Aeronautics and Space related to the Technology Committee of the Ministry of Science and Technology during from 1998 to 2000. He was a councilor of Saga University for 2002 and 2003. He also was an executive councilor for the Remote Sensing Society of Japan for 2003 to 2005. He is an Adjunct Professor of University of Arizona, USA since 1998. He also is Vice Chairman of the Science Commission "A" of ICSU/COSPAR since 2008 then he is now award committee member of ICSU/COSPAR. He wrote 37 books and published 570 journal papers. He received 30 of awards including ICSU/COSPAR Vikram Sarabhai Medal in 2016, and Science award of Ministry of Mister of Education of Japan in 2015. He is now Editor-in-Chief of IJACSA and IJISA. http://teagis.ip.is.saga-u.ac.jp/index.ht. 